\title{
Implantación en el laboratorio de un algoritmo para el diagnóstico diferencial de la proteinuria
}

\author{
M. D. ALBALADEJO OTÓN, M. M. GONZÁLEZ CUEVA, R. ÁLVAREZ LÓPEZ1', \\ P. MARTÍNEZ HERNÁNDEZ
}

Servicio de Análisis Clínicos y ${ }^{\prime}$ Servicio de Inmunología. Hospital Universitario Virgen

de la Arrixaca. El Palmar, Murcia

THE USE OF A SIMPLE ALGORITHM TO IMPROVE PROTEINURIA
DIAGNOSIS IN CLINICAL LABORATORIES

\section{RESUMEN}

Fundamento y objetivo: El hallazgo de proteinuria debe ir seguido de un diagnóstico diferencial para determinar su origen. Nuestro objetivo ha sido valorar los resultados de la implantación por el laboratorio de un algoritmo para el cribado y diagnóstico de la proteinuria.

Material y métodos: De un total de 30.718 orinas procesadas, se obtuvo una concentración de proteínas igual o superior a $30 \mathrm{mg} / \mathrm{dl}$ en 639, recomendándose el envío de una nueva muestra para confirmación y estudio diferencial de proteinuria. Se recibieron 207, a las que, además de las proteínas totales, se les cuantificaron creatinina, albúmina y alfa-1microglobulina, junto con los parámetros de la tira reactiva pseudoperoxidasa y esterasa leucocitaria. Estos resultados se incorporaron a un sistema experto (UPES y su aplicación Protis), que permite la diferenciación de hematuria, leucocituria y proteinuria, solicitando la medida de otras pruebas (IgG, alfa-2-macroglobulina, cadenas ligeras kappa/lambda) cuando es necesario.

Resultados: De las 207 peticiones recibidas para estudio de proteinuria selectiva, 39 fueron normales, 96 se clasificaron como glomerulopatía primaria, 26 como glomerulopatía secundaria y 5 como nefropatía túbulo-intersticial. Se pudo diferenciar el origen de la hematuria en 58 de estas orinas. Además, detectamos cadenas ligeras libres tipo kappa, confirmadas por inmunofijación, en una muestra de orina de un paciente con un proteinograma normal en suero.

Conclusión: Con el algoritmo propuesto, la información obtenida de una muestra de orina aumenta mucho, permitiendo la detección y diferenciación de la proteinuria y aportando sugerencias para la evaluación clínica del paciente.

PALABRAS CLAVE: Proteinuria. Diagnóstico. Algoritmo.

\section{ABSTRACT}

Background and objective: When proteinuria appears, a differential diagnosis must determine its origin. The object of this work has been to evaluate the results after the laboratory implantation of an algorithm for the screening and diagnosis of proteinuria.

Material and methods: From a total of 30,718 processed urines, a 30 $\mathrm{mg} / \mathrm{dl}$ or higher protein concentration was obtained in 639 , recommending a new sample to confirm and differenciate proteinuria. We received 207, to which total protein, creatinine, albumin and alpha-1-microglobulin were quantificated, together with pseudoperoxidase and leucocite esterase from dipstick. The results were introduced in an expert system (UPES and its application Protis), allowing differenciate hematuria, leucocituria and proteinuria and suggesting the assessment of other parameters, like IgG, alpha-2-macroglobulin, light chain kappa/lambda, when necessary.

Results: From 207 urinalysis assayed for selective proteinuria, 39 were normal, 96 were classified as primary glomerulopathy, 26 as secondary glomerulopathy and 5 as tubulo-interstitial nephropathy. A differential diagnosis of hematuria was made in 58 of these urines. Besides, kappa light chains were detected in a sample from a patient with a normal serum protein graph, which were confirmed by inmune fixation.

Conclusion: With the proposed algorithm, the information obtained from a urine sample increases substantially, allowing detection and differentiation of proteinuria and providing suggestions for the clinical evaluation of the patient.

KEY WORDS: Proteinuria. Diagnosis. Algorithm.

Albaladejo Otón MD, González Cueva MM, Álvarez López R, Martínez Hernández P. Implantación en el laboratorio de un algoritmo para el diagnóstico diferencial de la proteinuria. An Med Interna (Madrid) 2005; 22: 461-464.

\section{INTRODUCCIÓN}

El análisis de anormales y sedimento en orina es una de las pruebas más comúnmente solicitadas al laboratorio clínico, siendo de gran utilidad en el diagnóstico precoz de la enfermedad renal. Con frecuencia, ésta se acompaña del hallazgo ocasional de proteinuria en un análisis sistemático de orina, lo que puede preceder incluso en años a otros signos de enfermedad renal, como la elevación de creatinina plasmática. Además, la proteinuria es un factor de riesgo 
independiente para el desarrollo de enfermedad cardiovascular, relacionándose con la mortalidad, la morbilidad cardiovascular y el fracaso renal (1). También es un factor de riesgo para el desarrollo de enfermedad renal avanzada y su reducción es beneficiosa a largo plazo, ya que el exceso de proteínas plasmáticas ultrafiltradas es tóxico para las células epiteliales tubulares (2).

En los estados hipertensivos se producen alteraciones en la hemodinámica renal, que también pueden conducir a un daño de este órgano si no hay un buen control de las cifras tensionales. La microalbuminuria es un marcador precoz de afectación renal en la población general, aumentando su prevalencia en los pacientes hipertensos y cuando se asocia a otros factores de riesgo cardiovascular (3). No obstante, además de la excreción de albúmina, la de otras proteínas de bajo peso molecular, filtradas por el glomérulo y metabolizadas por las células del túbulo proximal, también puede reflejar la erosión producida en el riñón (4).

Por otra parte, la proteinuria puede tener un origen prerenal, como en el caso de la proteína de Bence-Jones, cuya detección precoz es importante, incluso a bajas concentraciones, para diagnosticar enfermedades linfoproliferativas y prevenir el fracaso renal en estos pacientes (5). A pesar de su importancia, la presencia de proteinuria no siempre indica un estado patológico, por lo que su hallazgo debe ir seguido de un diagnóstico diferencial para determinar su origen.

El objetivo del presente trabajo es valorar los resultados de la implantación por el laboratorio clínico de un algoritmo para el cribado y diagnóstico diferencial de la proteinuria según su origen y la presencia de hematuria adicional.

\section{MATERIAL Y MÉTODOS}

Se incluyeron un total de 30.718 orinas para realizar anormales y sedimento procedentes de pacientes de nuestra área sanitaria durante la primera mitad del año 2004. A todas las orinas simultáneas se les realizó un análisis sistemático mediante tira reactiva en un analizador de orinas Urisys 2.400 (Roche diagnostics). De ellas, 1.138 presentaron albúmina en la tira reactiva superior a $25 \mathrm{mg} / \mathrm{dl}(1.138 / 30.718=3,7 \%)$, y se les realizó la cuantificación de las proteínas totales en un analizador modular $\mathrm{P}$ (Roche diagnostics), mediante un ensayo inmunoturbidimétrico utilizando cloruro de bencetonio como agente precipitante, obteniendo una concentración de proteínas igual o superior a $30 \mathrm{mg} / \mathrm{dl}$ en $639(639 / 1.138=$ $56,1 \%)$.

Con estas 639 muestras seleccionadas se prosiguió enviando un informe al médico solicitante, indicando la oportunidad de mandar una nueva muestra de orina para confirmación y estudio diferencial de proteinuria. Se recibieron 207 nuevas muestras $(32,4 \%$ del total), a las que se les realizaron las siguientes pruebas: nueva tira reactiva de orina para valorar leucocitos y hematíes en el analizador Urisys 2.400 , proteínas totales, albúmina y creatinina en el modular P y alfa-1-microglobulina en un nefelómetro BN ProSpec (Dade Behring). En el caso de que estuviera indicado, se continuaba el estudio con ensayos adicionales: $\mathrm{IgG}$, alfa-2-macroglobulina e incluso cadenas ligeras de inmunoglobulinas kappa y lambda cuantificadas por nefelometría mediante el BN Prospec.
La estrategia diagnóstica aplicada consiste en cuantificar albúmina y alfa-1-microglobulina, como marcadores glomerular y tubular, respectivamente, además de proteínas totales y creatinina, esta última como indicador de la concentración de la muestra y frente a cuya masa referiremos los demás parámetros. La esterasa leucocitaria y la pseudoperoxidasa de la hemoglobina de la tira reactiva informan de la existencia de inflamación y hematuria. En estos casos es necesario medir la excreción de IgG y alfa-2-macroglobulina, para identificar la selectividad y el origen renal o post-renal de la proteinuria. Además, la disminución del índice albúmina/proteínas totales nos indica un posible origen pre-renal, como es el caso de la proteinuria de BenceJones, en cuyo caso se cuantifican cadenas ligeras kappa y lambda en la muestra. Un cociente kappa/lambda inferior a 1 ó superior a 3 indica la necesidad de realizar una inmunofijación, para descartar una gammapatía de origen monoclonal (6).

Sistemas expertos. El sistema experto UPES (Urine Protein Expert System) y su aplicación Protis (Dade Behring) está basado en una base de datos que contiene más de 500 patrones de excreción de pacientes con enfermedades conocidas, de los que se obtiene una base de conocimiento ampliada con diferentes flujos de trabajo considerando diversas situaciones clínicas. El patrón de excreción de las proteínas plasmáticas permite la diferenciación de hematuria, leucocituria y proteinuria, y asignarle una causa prerenal, renal o post-renal. El informe del paciente para el análisis de orina incluye el test de interpretación y dos gráficas opcionales que permiten la diferenciación entre glomerulopatía primaria, secundaria y nefropatía túbulo-intersticial y hematuria renal y post-renal, aportando sugerencias para la interpretación clínica de proteínas específicas que pueden apoyar el diagnóstico y mejorar el conocimiento clínico (7).

\section{RESULTADOS}

De las 207 peticiones recibidas para estudio de proteinuria selectiva, 39 fueron normales, 96 se clasificaron como glomerulopatía primaria, 26 como glomerulopatía secundaria, 5 como nefropatía túbulo-intersticial y 41 quedaron sin clasificar. En la tabla I se expresan la media y desviación estándar de la concentración de cada una de las proteínas cuantificadas en el grupo total y para cada uno de los grupos clasificados. 96 de las orinas estudiadas presentaron hematuria adicional a la proteinuria (46,3\% del total). El sistema también nos permitió clasificar el origen de la hematuria en 58 de estas orinas: $93,1 \%$ fueron de origen renal y $6,9 \%$ post-renales. En la tabla II se muestra la concentración de las proteínas cuantificadas en cada uno de estos grupos.

Del grupo de las 41 orinas sin clasificar, 19 lo fueron por presentar leucocituria y/o hematuria, 4 correspondieron a proteinuria post-renal y las 18 restantes a proteinuria pre-renal. Las 11 proteinurias pre-renales con hematuria fueron derivadas a estudio de hemoglobinuria y mioglobinuria, y a las otras 7 se le cuantificaron cadenas ligeras kappa y lamda. 2 de las orinas analizadas presentaron un cociente kappa/lambda superior a 3 y se les realizó una inmunofijación. Una de las muestras presentó una gammapatía con producción de cadenas ligeras libres tipo kappa. 
TABLA I

CONCENTRACIÓN DE PROTEÍNAS OBTENIDAS EN LOS DISTINTOS GRUPOS

\begin{tabular}{|c|c|c|c|c|c|c|}
\hline & $\begin{array}{l}\text { Creatinina } \\
(m g / d l) \\
\text { Media } \\
\text { D.E. }\end{array}$ & $\begin{array}{l}\text { Proteínas totales } \\
\text { (mg/g crea) } \\
\text { Media } \\
\text { D.E. }\end{array}$ & $\begin{array}{l}\text { Albúmina } \\
\text { (mg/g crea) } \\
\text { Media } \\
\text { D.E. }\end{array}$ & $\begin{array}{c}\text { Alfa-1-microg/obulina } \\
\text { (mg/g crea) } \\
\text { Media } \\
\text { D.E. }\end{array}$ & $\begin{array}{c}\lg G \\
\text { (mg/g crea) } \\
\text { Media } \\
\text { D.E. }\end{array}$ & $\begin{array}{c}\text { Alfa-2-macroglobulina } \\
\text { (mg/g crea) } \\
\text { Media } \\
\text { D.E. }\end{array}$ \\
\hline $\begin{array}{l}\text { Grupo total } \\
(\mathrm{N}=207)\end{array}$ & $\begin{array}{l}93,2 \\
54,5\end{array}$ & $\begin{array}{l}771,3^{*} \\
964,2\end{array}$ & $\begin{array}{c}345,2^{*} \\
426,0\end{array}$ & $\begin{array}{l}21,1^{*} \\
33,5\end{array}$ & $\begin{array}{l}43,4^{*} \\
83,3\end{array}$ & $\begin{array}{c}6,7 \\
20,8\end{array}$ \\
\hline $\begin{array}{r}\text { Crupo } 0 \\
(\mathrm{~N}=39)\end{array}$ & $\begin{array}{l}116,7 \\
66,2\end{array}$ & $\begin{array}{l}96,4 \\
53,9\end{array}$ & $\begin{array}{l}7,3 \\
6,0\end{array}$ & $\begin{array}{l}5,6 \\
1,7\end{array}$ & $\begin{array}{l}3,3 \\
0,4\end{array}$ & $\begin{array}{l}2,8 \\
2,4\end{array}$ \\
\hline $\begin{array}{l}\text { Crupo } 1 \\
(\mathrm{~N}=96)\end{array}$ & $\begin{array}{l}90,0 \\
47,5\end{array}$ & $\begin{array}{l}797,4^{*} \\
737,0\end{array}$ & $\begin{array}{c}456,7^{*} \\
416,3\end{array}$ & $\begin{array}{l}11,0 \\
10,0\end{array}$ & $\begin{array}{c}36,2^{*} \\
55,8\end{array}$ & $\begin{array}{l}3,5 \\
3,6\end{array}$ \\
\hline $\begin{array}{r}\text { Grupo } 2 \\
(\mathrm{~N}=26)\end{array}$ & $\begin{array}{c}57,0^{*} \\
28,1\end{array}$ & $\begin{array}{c}1293,6^{*} \\
1141,3\end{array}$ & $\begin{array}{c}703,0^{*} \\
585,0\end{array}$ & $\begin{array}{c}53,8^{*} \\
27,5\end{array}$ & $\begin{array}{l}93,1^{*} \\
129,1\end{array}$ & $\begin{array}{l}3,7 \\
3,1\end{array}$ \\
\hline $\begin{array}{c}\text { Crupo } 3 \\
(\mathrm{~N}=5)\end{array}$ & $\begin{array}{l}139,8 \\
88,6\end{array}$ & $\begin{array}{c}187,0^{*} \\
90,9\end{array}$ & $\begin{array}{c}25,8^{*} \\
20,2\end{array}$ & $\begin{array}{c}70,5^{*} \\
37,1\end{array}$ & $\begin{array}{l}12,8^{*} \\
14,6\end{array}$ & - \\
\hline $\begin{array}{r}\text { Crupo } 4 \\
(\mathrm{~N}=41)\end{array}$ & $\begin{array}{l}95,9 \\
53,2\end{array}$ & $\begin{array}{c}1027,0^{*} \\
1375,0\end{array}$ & $\begin{array}{c}187,6^{*} \\
199,6\end{array}$ & $\begin{array}{l}32,0^{*} \\
57,2\end{array}$ & $\begin{array}{l}70,4^{*} \\
116,9\end{array}$ & $\begin{array}{l}15,0^{*} \\
38,2\end{array}$ \\
\hline
\end{tabular}

Grupo 0= orinas normales; Grupo 1= glomerulopatía primaria; Grupo 2= glomerulopatía secundaria; Grupo 3= nefropatía túbulo-intersticial; Grupo 4= orinas no clasificadas. Valores de referencia: Creatinina: 90-300 mg/dl; Proteínas totales: 40-100 mg/g crea; Alfa-1-microglobulina: 0,1-14 mg/g crea; Albúmina: 1-20 mg/g crea; IgG: 0,1-10 mg/g crea; Alfa-2-macroglobulina: 0,1-10 mg/g crea. El símbolo * indica los parámetros que exceden los valores de referencia en cada grupo.

TABLA II

CONCENTRACIÓN DE PROTEÍNAS CLASIFICADAS EN FUNCIÓN DE LA PRESENCIA DE HEMATURIA

\begin{tabular}{|c|c|c|c|c|c|c|}
\hline & $\begin{array}{c}\text { Creatinina } \\
(m g / d l) \\
\text { Media } \\
\text { D.E. }\end{array}$ & $\begin{array}{l}\text { Proteínas totales } \\
\text { (mg/g crea) } \\
\text { Media } \\
\text { D.E. }\end{array}$ & $\begin{array}{c}\text { Albúmina } \\
\text { (mg/g crea) } \\
\text { Media } \\
\text { D.E. }\end{array}$ & $\begin{array}{c}\text { Alfa-1-microglobulina } \\
\text { (mg/g crea) } \\
\text { Media } \\
\text { D.E. }\end{array}$ & $\begin{array}{c}\lg G \\
\text { (mg/g crea) } \\
\text { Media } \\
\text { D.E. }\end{array}$ & $\begin{array}{c}\text { Alfa-2-macroglobulina } \\
\text { (mg/g crea) } \\
\text { Media } \\
\text { D.E. }\end{array}$ \\
\hline $\begin{array}{l}\text { Grupo sin hematuria } \\
(\mathrm{N}=111)\end{array}$ & $\begin{array}{l}95,0 \\
56,7\end{array}$ & $\begin{array}{l}534,4 \\
674,2\end{array}$ & $\begin{array}{l}255,2 \\
359,3\end{array}$ & $\begin{array}{l}18,5 \\
34,5\end{array}$ & $\begin{array}{l}23,8 \\
38,8\end{array}$ & - \\
\hline $\begin{array}{l}\text { Grupo con hematuria } \\
\text { renal }(\mathrm{N}=54)\end{array}$ & $\begin{array}{l}83,9 \\
39,4\end{array}$ & $\begin{array}{l}1067,0 \\
764,2\end{array}$ & $\begin{array}{l}627,4 \\
445,8\end{array}$ & $\begin{array}{l}19,3 \\
23,0\end{array}$ & $\begin{array}{l}53,9 \\
71,0\end{array}$ & $\begin{array}{l}3,5 \\
3,3\end{array}$ \\
\hline $\begin{array}{l}\text { Grupo con hematuria } \\
\text { post-renal }(\mathrm{N}=4)\end{array}$ & $\begin{array}{l}61,0 \\
35,3\end{array}$ & $\begin{array}{l}1754,8 \\
903,1\end{array}$ & $\begin{array}{l}556,3 \\
293,4\end{array}$ & $\begin{array}{l}72,8 \\
70,8\end{array}$ & $\begin{array}{l}223,7 \\
155,6\end{array}$ & $\begin{array}{l}26,7 \\
23,0\end{array}$ \\
\hline $\begin{array}{l}\text { Grupo con hematuria } \\
\text { no clasificada }(\mathrm{N}=38)\end{array}$ & $\begin{array}{c}104,7 \\
65,4\end{array}$ & $\begin{array}{c}932,6 \\
1580,2\end{array}$ & $\begin{array}{l}181,2 \\
401,5\end{array}$ & $\begin{array}{l}25,7 \\
34,5\end{array}$ & $\begin{array}{l}67,0 \\
139,9\end{array}$ & $\begin{array}{l}10,1 \\
34,4\end{array}$ \\
\hline
\end{tabular}

Valores de referencia: creatinina: 90-300 mg/dl; Proteínas totales: 40-100 mg/g crea; Alfa-1-microglobulina: 0,1-14 mg/g crea; Albúmina: 1-20 mg/g crea; IgG: 0,1-10 mg/g crea; Alfa-2macroglobulina: $0,1-10 \mathrm{mg} / \mathrm{g}$ crea.

\section{DISCUSIÓN}

Hoy en día, los sistemas analíticos generan una gran cantidad de resultados en el laboratorio. Mediante el uso de programas informáticos se simplifica el flujo de datos, permitiendo una mayor automatización del laboratorio y ofreciendo posibilidades de interpretación de los resultados para los clínicos. Con la estrategia diagnóstica propuesta, la información obtenida a partir de un análisis de orina aumenta notablemente, y esto mejora la calidad ofrecida por el laboratorio (8).

A partir de una muestra de orina simultánea, el algoritmo aplicado permite cuantificar y a la vez diferenciar la proteinuria, mediante la determinación de proteínas de distinto peso molecular. El patrón glomerular primario se caracteriza por proteínas totales bastante elevadas, con aumento de albúmina e $\mathrm{IgG}$, mientras que en el glomerular secundario también hay un aumento de alfa-1-microglobulina, y una disminución significativa de la creatinina. El patrón túbulo-intersticial presenta proteínas totales ligeramente elevadas, con predominio de alfa-1-microglobulina (Tabla I).

El grupo de orinas no clasificadas es un grupo heterogéneo, en el que se incluye la leucocituria, la hematuria post-renal y la proteinuria pre-renal. Un índice albúmina/proteínas totales inferior a 0,4 , en ausencia de otras alteraciones, nos alerta sobre una posible proteinuria de Bence-Jones, en cuyo caso se cuantifican cadenas ligeras libres kappa y lambda. En nuestro grupo de orinas remitidas para estudio de proteinuria diferencial detectamos cadenas ligeras libres tipo kappa, confirmadas por inmunofija- 
ción, en una muestra de orina. La proteína de Bence-Jones es el único producto biológico excretado en el 15-20\% de los procesos linfoproliferativos (9), y en nuestro caso el paciente presentaba un proteinograma normal en suero.

En el grupo de proteinuria con hematuria, la mayor concentración de alfa-2-macroglobulina se encontró en el grupo con hematuria post-renal, siendo esta macroproteína un marcador útil para su diagnóstico (Tabla II).

Así, la base de conocimiento aplicada nos permite la detección precoz de procesos inflamatorios y nefrotóxicos a partir de una única muestra de orina y mediante una técnica no invasiva. Lun y cols., al evaluar este sistema experto en una muestra de 146 niños diagnosticados de enfemedad renal, han comprobado su utilidad en el diagnóstico y diferenciación de enfermedades renales al compararlo con otras técnicas invasi-

\section{Bibliografía}

1. Kannel WB, Stampfer MJ, Castelli WP, Verter J. The prognostic significance of proteinuria: the Framingham study. Am Heart J 1984; 108: 1347-52.

2. Guder WG, Ivandic M, Hofmann W. Physiopathology of proteinuria and laboratory diagnostic strategy based on single protein analysis. Clin Chem Lab Med 1998; 36: 935-9.

3. Bonet J, Vila J, Alsina MJ, Ancochea L, Romero R. Prevalencia de microalbuminuria en la población general de un área mediterránea española y su asociación con otros factores de riesgo cardiovascular. Med Clin (Barc) 2001; 116: 573-4.

4. Newman DJ, Thakkar H and Gallagher H. Progressive renal disease: does the quality of the proteinuria matter or only the quantity? Clin Chim Acta 2000; 297: 43-54.

5. Pezzoli A, Pascali E. The clinical significance of pure Bence Jones proteinuria at low concentration. Am J Clin Pathol 1989; 91: 473-5. vas. Además, constataton mediante encuesta su alta valoración por parte de los clínicos solicitantes (10). Nosotros consideramos que la combinación de perfiles proteicos junto con otros parámetros del paciente y el cálculo de cocientes y fórmulas mediante programas informáticos, aporta sugerencias útiles para la interpretación clínica de los resultados, y puede ayudar a una correcta evaluación del paciente, con solo una muestra de orina.

\section{AGRADECIMIENTOS}

Queremos agradecer a $\mathrm{D}^{\mathrm{a}}$ Ana $\mathrm{M}^{\mathrm{a}}$ Hita López y $\mathrm{D}^{\mathrm{a}} \mathrm{M}^{\mathrm{a}}$ Carmen García Calatayud su cariño y ayuda en el análisis de las muestras.

6. Bergón E, Bergón M. Uso del cociente cadenas kappa/cadenas lambda en orina para el estudio de la proteína de Bence Jones. Quím Clín 1999; 18: $266-70$

7. Ivandic M, Hofmann W, Guder WG. The use of knowledge-based systems to improve medical knowledge about urine analysis. Clin Chim Acta 2000; 297: 251-60.

8. Regeniter A, Steiger JU, Scholer A, Huber PR, Siede WH. Windows to the ward: graphically oriented report forms. Presentation of complex, interrelated laboratory data for electrophoresis/immunofixation, cerebrospinal fluid, and urinary protein profiles. Clin Chem 2003; 49: 4150.

9. Fang LS. Light-chain nephropathy. Kidney Int 1985; 27: 582-92.

10. Lun A, Ivandic M, Priem F, Filler G, Kirschstein M, Ehrich JHH and Guder WG. Evaluation of pediatric nephropathies by a computerized Urine Protein Expert System (UPES). Pediatr Nephrol 1999; 13: 900-6. 